\title{
TRES RESPUESTAS A LOS ACUERDOS DE SAN ANDRÉS \\ Rodrigo Elizarrarás*
}

We're one, but not the same: Bono

\section{Introducción}

\section{Hoy en día resulta difícil encontrar} un Estado homogéneo. En su gran mayoría los países están constituidos por un conglomerado de culturas, lenguas, religiones, costumbres y razas. Según algunas aproximaciones, los 184 Estados independientes del mundo contienen más de 600 grupos lingüísticos y 5,000 grupos étnicos. Sin embargo, la herencia colonial con la que viven numerosos países del tercer mundo o la dominación política que ejerce un grupo sobre las minorías nacionales hacen muy complicada la conformación de una Constitución sensible a estas diferencias; pero sobre todo la composición de un Estado comprometido con la diversidad.

En Latinoamérica, una vez que se logró superar la tentación al genocidio y el exterminio, la tendencia común frente a la diversidad de etnias y culturas ha sido la de imponer un Estado fuerte y unificador. Donde se encontraron diferencias y 'desorden' se impuso la unidad del Estado-nación; fue la única solución que se creyó adecuada para organizar el rompecabezas étnico. Sin embargo, tras largos siglos de neutralización y exclusión de las minorías indígenas, han empezado a surgir voces que reclaman esta homogeneización como un claro atentado a sus identidades, a sus más intrínsecos valores como comunidades, pueblos o individuos. Tal es el caso que nos ocupa en este ensayo, el

* Ciencia Política, ITAM. 
RODRIGO ELIZARRARÁS

del movimiento zapatista en Chiapas, y en concreto el de las demandas políticas expresadas en los documentos conocidos como los Acuerdos de San Andrés.

La situación particular de estos acuerdos en el contexto socio-político-jurídico mexicano es, sin duda, excepcional dentro de una concepción liberal del Estado. La simple existencia de este documento ha desatado un intenso debate sobre la viabilidad de una serie de medidas encaminadas a la protección de culturas o pueblos en una evidente situación de desigualdad. De esta forma, la precaria democracia liberal mexicana se ha visto forzada a responder a dichas demandas, que se traducen en este debate como la concesión de 'derechos colectivos' atribuibles a las comunidades y pueblos indígenas.

Dichos derechos han encontrado un sinnúmero de objeciones dentro de la tradición político liberal, como son: que implican una ruptura con el único sujeto de la democracia liberal que puede ser poseedor de derechos y libertades, el individuo; y que contradicen los principios de igualdad que tienen todos los individuos frente al Estado y la ley. De hecho, no es extraño encontrar autores que los relacionan con políticas asociadas al nazismo, el Apartheid en Sudáfrica y a ciertas medidas segregacionistas adoptadas por algunos grupos de ortodoxia religiosa. Por cierto, hasta hace poco no existía una justificación válida para la aceptación de los derechos colectivos, pero parece que hoy algunos teóricos liberales empiezan a encontrar un espacio para los mismos sin necesidad de violentar los postulados fundamentales del liberalismo político.

Frente a este supuestamente nuevo reto del multiculturalismo al que se enfrentan los liberales de todo el mundo, han surgido las más variadas respuestas. La primera, corresponde a lo que se conoce como libertarismo, y pretende abordar el asunto como si se tratara de un simple tipo de pluralismo: con el silencio de la ley, la neutralidad del Estado y de las instituciones políticas. La segunda vertiente, el liberalismo estándar, se aleja un poco de la primera postura y pugna por una mayor conciencia de las diversas identidades y expresiones encontradas en una sociedad plural. Una respuesta reciente apunta hacia un mayor reconocimiento de la diversidad cultural, de la cultura como 
un valor primordial para el bienestar de los individuos, encontrando un lugar para este tipo de demandas y reconocimientos culturales dentro del liberalismo; a ella me referiré como liberalismo multiculturalista.

El presente ensayo es, de alguna forma, una breve discusión sobre la factibilidad de los derechos colectivos dentro de una concepción liberal del Estado. Sin embargo, esta discusión está acotada en varios sentidos. En primer lugar, el objeto central de la discusión son los supuestos 'acuerdos' entre el Gobierno mexicano y el Ejército Zapatista de Liberación Nacional, los Acuerdos de San Andrés. Otra limitación, frente al problema del multiculturalismo, es que abordaré el análisis de estos documentos a partir de tres enfoques canónicos del liberalismo, como son los de Chandran Kukathas, Jürgen Habermas y Will Kymlicka.

\section{San Andrés o de los derechos indígenas}

La Constitución mexicana, al igual que el resto de constituciones latinoamericanas, está inspirada en los principios y derechos de las llamadas 'Revoluciones liberales' del siglo XVIII. La influencia de la doctrina liberal en nuestras aspiraciones e instituciones políticas son quizá motivo de que resulte imposible encontrar algún tipo de referencia a la particularidad cultural, racial, sexual, linguística o étnica de los individuos; por el contrario, todos los hombres y mujeres son idénticos ante la ley, todos gozan de las mismas 'garantías individuales'. En este sentido, dentro de las normas jurídicas mexicanas no existía hasta hace pocos años ninguna mención al carácter pluricultural de la nación. El sujeto indígena era absorbido bajo la categoría totalizante de 'ciudadano mexicano". Como apunta Bartolomé Clavero: "Constitucionalmente, el espacio indígena es una zona de sombra, toda una constelación eclipsada", ya que no existe el reconocimiento legal a la identidad indígena como un sujeto per se de derechos diferenciados. Así, la diversidad

${ }^{1}$ Bartolomé Clavero, Derecho indigena y cultura constitucional en América, 1994, México, Siglo XXI, p. 115. 


\section{RODRIGO ELIZARRARÁS}

cultural de la nación se ha pretendido resuelta mediante el silencio de la ley, la igualdad y la unificación del sujeto jurídico.

En 1992, sin mayores obstáculos se realiza una modificación al artículo cuarto de la Constitución por la que se reconoce el carácter pluricultural de la nación mexicana. Esta medida que responde más a condiciones exteriores que a un consenso nacional de reconocimiento cultural, coincide curiosamente con la firma y adhesión de México a tratados internacionales, como el Convenio 169 de la Organización Internacional del Trabajo. ${ }^{2}$ Finalmente, la redacción del artículo modificado será:

La Nación mexicana tiene una composición pluricultural sustentada originalmente en sus pueblos indígenas: La ley protegerá y promoverá el desarrollo de sus lenguas, culturas, usos, costumbres, recursos y formas específicas de organización social, y garantizará a sus integrantes el efectivo acceso a la jurisdicción del Estado. En los juicios y procedimientos agrarios en que aquellos sean parte, se tomarán en cuenta sus prácticas y costumbres jurídicas en los términos que establezca la ley. ${ }^{3}$

Con esta reforma constitucional los pueblos e individuos indígenas por primera vez adquieren reconocimiento constitucional. Sin embargo, este primer paso aparentemente bienintencionado no ha derivado en ningún tipo de leyes, reglamentos o disposiciones al respecto, por lo que resulta un adorno más en la Constitución. Ha sido la opción menos problemática para reducir la tensión social sin tocar el cuerpo de la Constitución. ${ }^{4}$

${ }^{2}$ Al respecto, véase Díaz Polanco, Indigenous Peoples in Latin America, p. 83-8, donde expone que este tipo de reformas responde a dos tipos de presiones: la primera, de carácter interno, se refiere a los diferentes movimientos indígenas (organizaciones, guerrillas, partidos, etc.); la segunda, tiene que ver con los pronunciamientos y reconocimientos internacionales.

${ }^{3}$ Artículo Cuarto, Constitución Política de los Estados Unidos Mexicanos.

${ }^{4}$ Clavero, op. cit., p. 50. 
LOS ACUERDOS DE SAN ANDRÉS

El 16 de febrero de 1996, tras el levantamiento armado el EZLN y el Gobierno federal, en un supuesto mutuo consentimiento firman los llamados Acuerdos de San Andrés, que sentaban las bases para la creación de una ley de derechos y cultura indígenas, y expresaban por vez primera la intención de hacer explícito dentro de las leyes e instituciones nacionales el carácter pluricultural del país.

La propuesta general de los Acuerdos se reduce a crear "una nueva relación de los pueblos indígenas, la sociedad y el Estado", atendiendo a una histórica situación de desigualdad y discriminación de aquéllos, por lo que se requiere la construcción de una 'nueva política de Estado" que impulse el desarrollo y la justicia para los pueblos indígenas y que fortalezca su participación política en instituciones incluyentes.

Los Acuerdos ordenan las propuestas en seis grandes rubros: reconocimiento constitucional, derechos políticos, derechos jurídicos, derechos sociales, económicos y culturales. A su vez, se proponen cinco principios bajo los cuales se debe basar la nueva relación con el Estado: pluralismo, sustentabilidad, integralidad, participación y libre determinación. También prevén una serie de reformas constitucionales que se deben realizar a fin de que dichas propuestas sean congruentes con el sistema jurídico mexicano.

Según los Acuerdos de San Andrés el gobierno federal deberá asumir los siguientes compromisos:

1) Reconocer el estatuto constitucional de los pueblos indígenas y de su libre determinación, preservando la unidad nacional, con lo que podrán decidir su forma de gobierno interna y sus maneras de organización política, social, económica y cultural.

2) Ampliar la participación y representación política local y nacional, respetando sus tradiciones y fomentando un nuevo federalismo.

3) Garantizar el acceso pleno a la justicia, con reconocimiento y respeto a especificidades culturales y a sus sistemas normativos internos. 
RODRIGO ELIZARRARÁS

4) Impulsar políticas culturales nacionales y locales para la promoción de las actividades culturales indígenas.

5) Asegurar la educación y capacitación, respetando las tradiciones y saberes propios. La educación que imparta el Estado debe ser intercultural. Además se incluyen tres incisos que garantizan las necesidades básicas (alimentación, salud y vivienda), el impulso a la producción y el empleo, y los derechos de los emigrantes.

Después de las negociaciones entre el gobierno federal y el EZLN y de la firma de los Acuerdos de San Andrés, el gobierno federal decidió desconocer dichos acuerdos, provocando una separación inmediata con el grupo guerrillero y, en consecuencia, la suspensión de las negociaciones. Por su parte, el Congreso de la República tiene pendiente la discusión de tres propuestas sobre legislación en materia indígena. ${ }^{5}$ Hasta el momento la situación política y legal de los indígenas sigue en un proceso de estancamiento que no verá solución en el corto o mediano plazo, mientras que la situación social en el Estado de Chiapas continúa deteriorándose, y el silencio de ambas partes hace que la situación cotidiana se vuelva fastidiosa.

En México el debate sobre la situación indígena surgido a partir de la existencia (y las sucesivas discrepancias) de los Acuerdos de San Andrés se ha reducido esencialmente a la aceptación de dichos documentos, que para muchos implica casi automáticamente la solución del problema étnico en Chiapas. No existen antecedentes que hayan colocado en el centro del debate nacional la cuestión sobre el destino y situación de los pueblos indígenas con la magnitud y alcance con que este movimiento lo ha logrado. Por esto los Acuerdos de San Andrés constituyen el objeto central de análisis para discutir la situación de las minorías étnicas en este país, así como para evaluar la

${ }^{5}$ En la pila de materias pendientes de la legislatura actual están tres iniciativas en materia de derecho indígena, una propuesta por el ejecutivo, una del Partido Acción Nacional y una del Partido Verde Ecologista Mexicano. 
LOS ACUERDOS DE SAN ANDRÉS

congruencia de sus postulados políticos con los principios implícitos de una democracia liberal.

\section{Kukathas o de la neutralización de las diferencias}

Dentro de la corriente liberal existen divergencias en el tratamiento de ciertos problemas contemporáneos, y las numerosas respuestas al problema del multiculturalismo constituyen un claro ejemplo. Kukathas lo aborda de la misma manera que si se tratara de cualquier pluralismo -religioso, ideológico, de género, etc. $-{ }^{6}$ con lo que se muestra como un exponente de lo que podríamos denominar libertarismo.

Para este autor el multiculturalismo no representa un problema, precisamente porque la posición del liberalismo es la de resistir a este tipo de demandas: "El liberalismo no tiene ningún problema con la pregunta de si se debe en virtud de la dignidad humana reconocer identidades individuales o las identidades de los grupos. El liberalismo no está preocupado en otorgar reconocimientos a ninguno de los dos. No ofrece ningún reconocimiento en absoluto."7 En otras palabras, la acitud del liberalismo frente a demandas de reconocimiento de especificidad cultural de un grupo o comunidad es no hacer nada. Además de que "la razón por la cual el liberalismo no tiene ningún problema con el multiculturalismo es porque el liberalismo es en sí, fundamentalmente, una teoría sobre multiculturalismo". ${ }^{8}$ Con lo que Kukathas defiende al liberalismo como la respuesta más plausible al hecho de la diversidad moral, religiosa y cultural del mundo moderno.

${ }^{6}$ A lo que esto se refiere es que esta vez están en juego una serie de diferentes concepciones del bien, de lo justo, de la política y, en general, una diferente concepción de la vida pública y privada.

${ }^{7}$ Chandran Kukathas, "Liberalism and Multiculturalism; The Politics of Indifference", en Political Theory, oct. 1998, vol. 26, n 5, p. 691.

${ }^{8}$ Idem, p. 690. 
RODRIGO ELIZARRARÁS

Según este autor, el liberalismo debe seguir siendo the politics of indifference, indiferente a los grupos y comunidades a las que pertenecen los individuos:

Los individuos son libres de formar grupos o asociaciones, de continuar sus asociaciones con los grupos a los que se han unido, o en los que han nacido. El liberalismo no tiene ningún interés en estos vínculos - culturales, religiosos, étnicos, lingüísticos, etc.- que la gente pueda tener [...] no tiene proyectos colectivos, no expresa preferencias de grupos, y no promueve intereses ni individuos particulares. Su única preocupación es la de mantener el marco jurídico que permita interactuar pacíficamente a grupos e individuos. ${ }^{9}$

Esta postura -próxima a la de teóricos libertarios como Nozick- ${ }^{10}$ como la de la mayoría de los liberales, no otorga valor a ninguna reivindicación cultural. Kukathas argumenta que las identidades culturales no son fáciles de identificar, y que resulta muy complicado y peligroso derivar derechos de las mismas. Además, observa que las culturas no son estáticas sino mutables y adaptables, dependiendo de los factores políticos, económicos y sociales en las que se desarrollen. Tomando como ejemplo el caso de diferentes grupos de migrantes chinos, que varían sus prácticas económicas dependiendo del país al que emigran, llega incluso a afirmar que la identidad cultural es una 'cuestión de elección ${ }^{11}$ y no un valor determinante e intrínseco de cada persona. Este ejemplo le parece suficiente para sustentar que la 'cultura' de estos grupos es mutable, en función a los diferentes factores y contextos

${ }^{9}$ Idem, p. 691.

${ }^{10}$ Kukathas llega a exponer en varias ocasiones definiciones nozickianas del Estado, afirmando que la única preocupación de éste debe ser la de preservar el orden entre los grupos y entre los individuos.

${ }^{11}$ Chandran Kukathas, "The Idea of a Multicultural Society", en Kukathas (ed.), Multicultural Citizens: the philosophy and politics of identity, 1993, Leonard Nsw, Center of Independent Studies, p. 23-4. 
en los que se desarrollan. ¿Acaso el cambio en las preferencias políticas, en las costumbres religiosas o económicas de algunos grupos indígenas en Chiapas son efectivamente un cambio cultural, a pesar de que conserven el resto de las características que definen a una cultura, como un conjunto de valores, historia común, creencias, tradiciones, instituciones y lengua? Es indudable que una parte de sus tradiciones ha sido sustituida o modificada, lo que no significa que por ello hayan dejado de formar parte de una misma cultura. Kukathas confunde el cambio en una costumbre de una tradición debido a circunstancias particulares, con un cambio en el conjunto de costumbres, valores y tradiciones; lo cual sólo es posible, desde mi modo de verlo, por un suceso tan radical como una conquista o una revolución cultural.

Como Kukathas no ofrece ninguna definición de cultura, le resulta fácil afirmar que un cambio en las prácticas económicas, religiosas o políticas son suficientes para hablar de un cambio cultural. Si esto fuera así, observaríamos que a partir de la revolución industrial los ingleses habrían experimentado un cambio cultural muy fuerte, no obstante conservar el resto de sus instituciones intactas (lengua, religión, historia, etc.).

Por otro lado, la 'neutralidad liberal' de Kukathas ha recibido numerosas objeciones. Para algunos críticos esa pretendida neutralidad no es sino reflejo de los valores y costumbres de la dominante la cultura liberal. ${ }^{12}$ Dice James Tully que en un Estado multicultural para lograr un acuerdo en verdad democrático e incluyente es necesario abrir el diálogo a todas la expresiones culturales. Kukathas responde que esa interpretación es inadecuada y errónea, porque el liberalismo es más que una simple expresión cultural.

Kukathas no claudica en su defensa del liberalismo como teoría del pluralismo, y ofrece dos razones principales: la primera consiste en que el pluralismo se puede defender con mayor efectividad si se cuenta

${ }^{12}$ Para una exposición sobre un constitucionalismo multicultural, véase James Tully, Strange Multiplicity, Constitutionalism in the age of diversity, 1998, EU, Cambridge Press. 
con instituciones que protejan los derechos y libertades de los individuos en lugar de sus intereses, ${ }^{13}$ dado que las preferencias personales cambian. Por lo tanto, las instituciones políticas deben diseñarse para defender los derechos y libertades individuales, evitando la injerencia de intereses sectarios; es decir, deben permitir aflorar las diferencias, pero en ningún momento determinar la conformación de una sociedad. ${ }^{14}$

Respecto al reconocimiento de la identidad indígena, este autor podría estar de acuerdo con algunos de los derechos incluidos en los Acuerdos de San Andrés, como los de representación, que únicamente demandarian algunos reajustes institucionales con el fin de dar mayor representatividad a las autoridades indígenas. Asimismo, con los derechos de pleno acceso a la justicia, que son parte fundamental de un Estado de derecho, y para el caso exigen ciertas modificaciones de instituciones y leyes con el fin de evitar un trato desigual entre los ciudadanos. Pero en ningún momento estaría de acuerdo con un orden jurídico alternativo al establecido, puesto que de implicaría la intromisión de otros intereses y costumbres.

Su segunda razón consiste en restringir las cuestiones culturales a una arena privada, pues si se llevan a la discusión pública se corre el riesgo de que las minorías pierdan sus derechos, debido a que nuevamente la mayoría impondría sus intereses en la sociedad. Por lo mismo, insiste en que el pluralismo se logra mejor tratando de preservar normas de tolerancia y respeto por las libertades individuales, que intentando conformar la sociedad de acuerdo con preferencias e intereses de los grupos existentes.

Tampoco aceptaría una defensa de los derechos de promoción de manifestaciones culturales de los pueblos como la que sostenęmos, pues piensa que la cultura debe encontrar caminos para su desarrollo que no dependan de las energías del Estado. Kukathas estaría de

${ }^{13}$ En la visión de este autor, hay un evidente rechazo a lo que él llama las soluciones de 'grupos de interés' (group participation approach); ver Kukathas, "Liberalism and Multiculturalism", en Political Theory.

${ }^{14}$ Kukathas, "The Idea of a Multicultural Society", p. 27. 
acuerdo en que la supervivencia cultural no puede reclamarse como un derecho, es algo que no puede garantizarse: "El estado no puede entrometerse en tratar de determinar qué culturas deben prevalecer y cuáles han de desaparecer, o de transformarse."15

En cuanto a las demandas de derechos de educación, capacitación y provisión de las necesidades básicas, son aún menos probables de encontrar aceptación por este tipo de liberalismo, pues no existe una razón para garantizar estos derechos especialmente a los indígenas. De existir un compromiso estatal con la educación, tema que no está especificado por este autor, sería con toda la sociedad, sin exclusiones ni preferencias. Apegándonos a los argumentos de Kukathas, impulsar la producción y el empleo no deberían ser funciones del Estado, sino cuestiones que se regulen por otros mecanismos. Además no resulta justificable porqué deben ser aseguradas para ciertos grupos y no para la sociedad en su conjunto.

Pero entonces ¿qué sugeriría que debe hacer el gobierno mexicano frente a estas demandas? ¿Se podría resistir a las mismas desde una justificación válida? Kukathas respondería que no es necesario reconocer estas demandas para alcanzar puntos como los de tolerancia y pluralidad. Además resulta peligroso este tipo de reconocimientos, ya que otros grupos podrían emprender reivindicaciones semejantes desencadenando un 'efecto dominó' difícil de controlar. ${ }^{16}$ Kukathas hace énfasis en la necesidad de canalizar este tipo de requerimientos mediante mecanismos legítimos dentro del liberalismo, y ofrece algunos ejemplos de soluciones que no implican modificaciones de las normas.

Si el problema es la paz, dice, las instituciones políticas pueden desarrollar arreglos (explicit power-sharing) entre grupos étnicos o religiosos. Propone la conformación de partidos políticos incluyentes, de fórmulas electorales más equitativas u otro tipo de mecanismos

${ }^{15}$ Kukathas, "Liberalism and Multiculturalism", p. 694.

${ }^{16}$ Kukathas, "Are there any Cultural Rights?", en Ian Shapiro y Will Kymlicka, (eds.), Ethnic and Group Rights, 1997, NY, New York University Press, p. 236. 


\section{RODRIGO ELIZARRARÁS}

integradores, que fomenten la participación de las minorías dentro de un marco de tolerancia y equidad entre los diferentes grupos que conforman la sociedad, evitando en todo momento los privilegios de una colectividad particular.

Debido a que las políticas de multiculturalismo pronto se convierten en espacios de grupos de interés, y que es inadmisible permitir que la sociedad se conforme mediante intereses particulares, su recomendación es eludir este tipo de reconocimiento del debate político, lo cual significa que el autor estaría en total desacuerdo con el primer punto de las demandas indígenas de los Acuerdos: su reconocimiento constitucional. Quizá gran parte de los puntos que exigen los pueblos indígenas sería alcanzable prescindiendo del énfasis cultural o étnico en la solución a problemas estructurales del sistema político mexicano; no obstante esta consideración, según este teórico caben algunas objeciones:

La primera es que estrictamente no existe un estado neutral, dado el carácter histórico de las instituciones de cada estado. Ciertamente, dice, que así como un edificio necesariamente tendrá un color, las instituciones poseerán especificaciones particulares que guardan más relación con los procesos históricos y las circunstancias que con su funcionalidad.

92 La segunda objeción, es que este tipo de liberalismo no es suficiente. El estado liberal comprometido con el florecimiento y la supervivencia de la nación y su cultura común debería perseguir algunos objetivos colectivos tratando de lograr una sociedad armoniosa y cohesionada, pero en un estado que se involucre en la construcción cultural de la nación, las minorías demandarán una parte en dicha tarea, por lo que estas leyes en vez de cohesión causarán disenso.

Finalmente, Kukathas admite que las actuales demandas de reconocimiento de diferentes grupos en una sociedad son un reto para las instituciones liberales. Al igual que otros teóricos, se pregunta qué tipo de arreglos e instituciones deben gobernar una sociedad multicultural sin poner en riesgo los postulados centrales del liberalismo. Sin embargo, salvo su ya conocida posición sobre la tolerancia y la defensa de la libertad individual, no es claro en su propuesta. No permite mayores interpretaciones sobre los Acuerdos, salvo una negación inmediata 
LOS ACUERDOS DE SAN ANDRÉS

simplemente por el hecho de ser demandas culturales provenientes de minorías. Su postura sobre cuestiones del tipo ha sido que "la diversidad debe ser acomodada y las diferencias toleradas; que una unidad social más completa, marcada por una cultura común y uniforme que integre y armonice los intereses del individuo y la comunidad es imposible e indeseable; que la división, el conflicto y la competencia estarán siempre presentes en la sociedad, y la tarea de las instituciones políticas es disminuir, atenuar una condición imposible de curar". ${ }^{17}$ Sostiene que el liberalismo ofrece la oportunidad de convivir dentro del mismo espacio a las más diversas manifestaciones culturales, religiosas, ideológicas, etcétera, no porque las leyes les otorguen un reconocimiento sino al contrario, precisamente debido al silencio de la ley.

\section{Habermas o del diálogo democrático}

Habermas interviene en el debate sobre multiculturalismo en respuesta a posiciones tan polarizadas como las de liberales a favor de la autonomía individual y las comunitaristas, expresadas principalmente por Charles Taylor. Para este autor, representante de un liberalismo 'estándar', la solución a los movimientos en busca de un reconocimiento de sus diferencias no está en la garantía de autonomía y libertad individual ni en la superposición de la comunidad por encima de las voluntades de los individuos. Ambos mundos son prácticamente inconciliables y se carece de respuesta satisfactoria a estos movimientos desde el liberalismo, pero es necesario conservar el respeto a los principios elementales de esta doctrina, así como las demandas de reconocimiento de las minorías, sin necesidad de recurrir a modelos normativos alternativos.

Consciente de la construcción extremadamente individualista que el sistema de derechos tiene bajo el liberalismo, Habermas se pregunta si es posible compaginar la base de dicha teoría con las luchas en

${ }^{17}$ Kukathas, "Liberalism and Multiculturalism", p. 690. 


\section{RODRIGO ELIZARRARÁS}

busca de reconocimiento, que amenazan la articulación y afirmación de identidades colectivas.

Citando a Amy Gutman, Habermas está interesado en comprobar si a partir del respeto por las identidades individuales de cada persona (autonomía privada) se puede derivar el respeto por las diferentes concepciones, actividades y prácticas de los individuos (autonomía pública), o si ambas reivindicaciones son incompatibles.

Para este teórico liberal, la Constitución puede concebirse como un proyecto histórico que cada generación de ciudadanos decide continuar. Este proyecto no es inamovible sino que las leyes se han de interpretar a la luz de nuevos problemas, necesidades, demandas e intereses. Como hemos mencionado, la concepción liberal del derecho presupone la noción tanto de derechos individuales como de sujetos individuales poseedores de éstos; pero, según Habermas, estos sujetos sólo podrían concebirse a sí mismos como autónomos si a la vez que destinatarios pueden verse como autores de las leyes. Y los autores serán libres solamente en tanto que participantes en un proceso legislativo regulado y ejecutado de manera que todos y cada uno puedan decir que las leyes y acuerdos derivados del mismo cuentan con su aceptación racional. En términos normativos, lo que Habermas está diciendo es que no puede existir un estado constitucional sin democracia. ${ }^{18}$

Lo anterior da una estocada mortal al sistema político mexicano, dado que la construcción y desarrollo de éste tuvo un contexto completamente antidemocrático, que derivó en la construcción de un supuesto Estado constitucional con mecanismos y prácticas más autoritarios que democráticos, que impusieron a toda costa la creencia en una identidad común y en la unidad nacional constituida por una federación de estados, igualmente carentes de identidad.

En estos momentos que la democracia empieza a filtrarse entre las instituciones políticas es oportuno y urgente reformular el 'contrato'

${ }^{18}$ Véase Jürgen Habermas, "Struggles for Recognition in the Democratic Constitutional State", en Charles Taylor et al., Multiculturalism: Examining the Politics of Recognition, 1994, Princeton NJ, Princeton University, p. 122. 
estatal, incluyendo tanto viejas como nuevas voces en su dinámica deliberativa y abriendo espacios para que quienes permanecen ajenos a la actual esfera pública sean incluidos y puedan sentirse autores de las leyes que los gobiernan.

Me parece que, en principio, Habermas avalaría tanto al movimiento zapatista de Chiapas como a los posteriores Acuerdos de San Andrés. Se trata de una manifestación que recurre a una vía armada y fuera de los cauces de la ley para exhibir el estado de segregación y aislamiento al no encontrar procedimientos democráticos y legales que lo hicieran posible. De haber existido un Estado constitucional democrático muy probablemente este problema hubiera encontrado otro camino.

Habermas piensa que interpretada apropiadamente la teoría de derechos de ninguna manera será ciega a las diferencias sociales y culturales. Como ya mencionaba, se trata de hacer una conexión necesaria entre democracia y Estado:

La ceguera de una lectura selectiva se desvanece una vez que asumimos que adscribimos a los poseedores de derechos individuales una identidad que es concebida de manera intersubjetiva. Las personas y las personas legales también, se hacen individuales sólo a través de un proceso de socialización. Una teoría de derechos correctamente entendida requiere de una política de reconocimiento que proteja la integridad de los individuos en el contexto de vida en que su identidad se forma. Esto no requiere un modelo alternativo que pueda corregir el diseño individualista del sistema de derechos a través de otra perspectiva normativa. Todo lo que se necesita es la actualización consistente del sistema de derechos. ${ }^{19}$

Con esto Habermas se desliga tanto de las posiciones individualistas exacerbadas de algunos liberales como de los postulados comunita-

${ }^{19}$ Idem, p. 113. 
RODRIGO ELIZARRARÁS

ristas, pero no deja claro qué entiende por actualización del sistema de derechos, que suponemos señala su constante revisión.

Habermas se manifiesta entonces a favor de un justo medio entre la identidad privada y la pública. Las leyes deben tomar en cuenta la socialización de los individuos para un mejor desenvolvimiento de sus vidas; $y$ advierte que en la medida en que las políticas estén enfocadas a salvaguardar la autonomía privada de los individuos, dejando de lado su conexión con una autonomía pública de los ciudadanos, las políticas de derechos oscilarán absurdamente entre los polos de un paradigma liberal, esto es, entre posturas de tipo lockeano y partidarias de un Estado benefactor.

La primera cuestión del multiculturalismo es sobre la neutralidad ética de la ley y la política, pues los juicios éticos no pueden ser formulados desde la visión particular de un grupo o persona que determine lo que es bueno para todos. En este sentido Habermas piensa que el liberalismo no es ciego ni neutral ante problemas morales o políticos, puesto que reconoce que el proceso de construcción de las leyes en parte está determinado por los valores y metas de una sociedad. "Las cuestiones éticas también entran en las deliberaciones y justificaciones

96 de las decisiones legislativas." ${ }^{20}$ De tal forma, que cada sistema legal no sólo es reflejo del contenido universal de los derechos básicos, sino también expresión de un particular modo de vida. Por ello, la apertura de un diálogo en este proceso democrático obedece a que lo que legitima las demandas no es la neutralidad de la ley sino la evidencia de que cada comunidad legal y cada proceso democrático para actualizar los derechos básicos inevitablemente se encuentran permeados por la ética. Viéndolo así, lo que cobra real importancia es el hecho de que tales decisiones dependen de la composición de la ciudadanía de un Estado-nación. Y si un Estado como el mexicano es plural, entonces será preciso posibilitar la participación de todos los sistemas de creencias, valores y costumbres que existen en su seno.

${ }^{20}$ Idem, p. 125. 
Entonces, la salvaguarda de la autonomía privada de ciudadanos con idénticos derechos debe activarse conjuntamente con su autonomía de ciudadanos de una nación. Cualquier versión liberal que no lo considere así, no entenderá la universalización de los derechos básicos como una igualación de las diferencias culturales y sociales. "Un orden legal es legítimo cuando salvaguarde la autonomía de todos los ciudadanos en el mismo grado." ${ }^{21}$ El liberalismo que privilegia la individual falla en reconocer que la autonomía privada y la pública tienen la misma prioridad. "Los individuos no pueden alcanzar el disfrute de libertades individuales iguales a menos que, ejercitando conjuntamente sus autonomías como ciudadanos, lleguen a un entendimiento claro sobre qué intereses y criterios están aceptados y en qué medida las cosas iguales serán tratadas como iguales y las cosas desiguales serán desiguales en cada caso particular." 22

Las demandas por respeto más que para equilibrar las condiciones de vida están dirigidas para proteger la integridad de las tradiciones y formas de vida en las que los miembros de ciertos grupos discriminados puedan reconocerse mutuamente. Por lo general esta falta de reconocimientos se halla reforzada por actitudes de discriminación.

Habermas concluye en que si una lectura 'selectiva' de una teoría de derechos es corregida para incluir un modelo de derechos básicos democrático como el que él propone, no hay necesidad de contrastar un liberalismo extremo, como el de Kukathas, con un modelo alternativo que introduce la noción de derechos colectivos, ajenos a esta doctrina, como proponen los comunitaristas. ${ }^{23}$

En otras palabras, lo que Habermas diría es que no es necesario construir un sistema alternativo de derechos que garantice el respeto cultural y las diferencias substanciales de los modos de vida de los pueblos, es decir, no serían necesarias las modificaciones que los Acuerdos de San Andrés demandan. En su lugar sería recomendable abrir el

${ }^{21}$ Idem, p. 121.
${ }^{22}$ Idem, p. 113.
${ }^{23}$ Idem, p. 116. 
RODRIGO ELIZARRARÁS

sistema de derechos a una mecánica democrática, así como procurar el respeto tanto de las individualidades como de las particularidades colectivas de los grupos.

En el caso específico de los acuerdos de San Andrés, estaría de acuerdo en que un énfasis excesivo en lo comunitario no sería la solución para el mejor cumplimiento de las demandas de estos grupos. En todo caso sería más adecuado abrir espacios y formar instituciones democráticas sensibles y abiertas al reconocimiento de estas diferencias. Es importante mantener el sistema de derechos con base en los individuos, pero tomando en cuenta sus contextos culturales y abriendo el debate a toda clase de visiones éticas de lo bueno, lo justo y lo deseable para cada uno de los miembros del Estado.

Habermas subraya la importancia del discurso y de la discusión sobre las concepciones comunes del bien y los deseos de vida que se quieren llevar a cabo. Mediante estas discusiones, los interlocutores pueden establecer la manera en que desean entenderse en tanto que ciudadanos de una república, habitantes de una región y herederos de una cultura específica; qué tradiciones desean conservar y cuáles no, qué relación mantendrán con su historia, sus semejantes, la naturaleza o la instancia que fuera. ${ }^{24}$

Su postura sobre el respeto y protección a culturas particulares es un tanto ambigua, sin embargo, en su crítica a Taylor y otros comunitaristas que reclaman restricciones a derechos básicos a efectos de preservar culturas en peligro de desaparecer, parece manifiesta su oposición a esta defensa.

\section{Kymlicka o rompiendo con la tradición liberal}

A diferencia de los autores expuestos anteriormente e incluso de la gran mayoría de intelectuales liberales, Kymlicka se pregunta por la relevancia de la cultura dentro de una teoría liberal de la justicia. Está interesado en descubrir cómo responden los pensadores liberales a la pluralidad ${ }^{24}$ Idem, p. 125. 
cultural. Una de las corrientes más comunes dentro del liberalismo concibe la libertad de asociación a todos los individuos y grupos dentro de una sociedad sin necesidad de reconocimiento legal o constitucional, dejando el papel de la cultura como una cuestión de ingreso a un club; sin embargo, el autor de Ciudadanía multicultural observa que esto no siempre fue así, y que es deseable y posible hacer una defensa de los derechos de las minorías desde el liberalismo.

Apoyándose en liberales como John S. Mill, que no necesariamente se oponen a estas defensas y en pensadores que durante el siglo XIX y principios del XX desde esta corriente filosófica no ignoraron los problemas con la cultura o la nacionalidad, este teórico canadiense está seguro de encontrar un espacio para los derechos de las minorías dentro del liberalismo. Es erróneo pensar que los liberales "siempre se han opuesto al reconocimiento político y al respaldo de la etnicidad y la nacionalidad, y que las reivindicaciones a favor de los derechos diferenciados en función del grupo para grupos culturales son una reciente desviación iliberal de una práctica liberal largamente establecida". ${ }^{25}$ Kymlicka se propone demostrar que la defensa de los derechos de las minorías constituye un componente legítimo de la tradición liberal, sustentando la idea de que para las culturas minoritarias una concepción universal de los derechos individuales resulta incompleta y requiere complementarse mediante la institución de derechos 'diferenciados en función de grupo'. Sin embargo, reconoce que hace falta explicar cómo es posible alcanzar la coexistencia de estos derechos con los principios de libertad individual, democracia y justicia social.

Según este autor, la actual aversión a los derechos colectivos en primer lugar se debe al temor del surgimiento de nuevos nacionalismos que puedan mermar la unidad y cohesión del Estado, y en segundo, a evitar protecciones o concesiones selectivas que atenten contra el principio de igualdad: "Los liberales temen que cualquier tipo de desviación del principio estricto de la igualdad de derechos para todos los individuos sea el primer paso hacia el apartheid, hacia un sistema donde

${ }^{25}$ Will Kymlicka, Ciudadania multicultural, 1996, Barcelona, Paidós, p. 78. 
RODRIGO ELIZARRARÁS

algunos individuos sean vistos como ciudadanos de primera clase y otros como de segunda clase, en virtud de sus afiliaciones étnicas o raciales." ${ }^{26}$ Contra esto, Kymlicka argumenta que:

La noción de respeto a las personas como miembros de una cultura, basada en el reconocimiento de la importancia del bien primario de la pertenencia cultural, no es, por consiguiente, una defensa iliberal. No dice que la comunidad es más importante que los individuos que la componen, o que el Estado debe imponer la mejor concepción de una buena vida a sus ciudadanos con tal de preservar la pureza de la cultura, o cualquiera de esas cosas. El argumento simplemente dice que la pertenencia cultural es importante para alcanzar nuestros intereses esenciales en una vida buena... y que dichas consideraciones sobre tal membresía es una parte importante para tener consideraciones iguales sobre los intereses de cada miembro de la comunidad. ${ }^{27}$

Kymlicka propone superar el que considera debate absurdo sobre 100 la primacía del individuo o de la comunidad y se declara en contra de la categoría de los 'derechos colectivos ${ }^{\text {"28 }}$ porque, dice, es un término muy amplio, y en su lugar propone la de 'derechos diferencia-

${ }^{26}$ Will Kymlicka, Liberalism, Community and Culture, 1995, EU, Oxford Press, p. 144.

${ }^{27}$ Kymlicka, op. cit., p. 166-168. Kymlicka defiende estos derechos basándose en una valoración de la cultura en términos rawlsianos, esto es que la cultura es un valor primordial para ciertas sociedades o grupos y que ésta debe tomarse en cuenta. Para Kymlicka el argumento de Rawls de la necesidad de la libertad como un bien primario, es también el argumento para defender a la pertenencia cultural como un bien primario. De esta manera debe reconocerse a la estructura cultural como un 'contexto de elección', desde el cual se determinan lo que realmente queremos de nuestras vidas.

${ }^{28}$ Para una detallada exposición de los diferentes tipos de derechos colectivos, véase "Classifying Cultural Rights", en Shapiro y Kymlicka, Ethnicity and Group Rights, 1995. 


\section{LOS ACUERDOS DE SAN ANDRÉS}

dos en función de grupo', de los que reconoce tres tipos: los de autogobierno, los poliétnicos y los de representación política. Aquí sólo consideramos los derechos de autogobierno y de representación política, pues son los que hallamos aludidos en los documentos de San Andrés y prescindiremos de los poliétnicos por tratarse de derechos referidos a minorías resultantes de inmigraciones masivas, que es una situación ajena. ${ }^{29}$ Sobre estos últimos sólo comentaremos que según Kymlicka estos derechos no son necesariamente otorgables a ciertas colectividades, por el contrario, dado que en la mayoría de los casos "no tienen que ver con la primacía de las comunidades sobre.los individuos, sino que más bien se basan en la idea de que la justicia entre grupos exige que a los miembros de grupos diferentes se les concedan derechos diferentes". ${ }^{30}$ Esta postura resulta adversa a las demandas zapatistas expresadas en los documentos de San Andrés, puesto que enfatizan la primacía de la comunidad.

Con respeto a los otros, que nos interesan en relación con los Acuerdos de San Andrés, sostiene Kymlicka:

1) Derechos de autogobierno: implican cierto grado de autonomía política o jurisdiccional territorial para asegurar el pleno y libre desarrollo de las culturas, con el fin de alcanzar sus propios intereses. Ejemplo de esto es el federalismo, que reparte poderes y responsabilidades entre el gobierno central y las entidades federativas.

${ }^{29}$ Kymlicka hace una diferencia entre lo que llama los Estados multinacionales, aquellos donde algunas naciones existen de manera original, y los Estados poliétnicos, conformados básicamente por las fuertes inmigraciones a esos países; existen Estados que pertenecen a ambas categorías. Los Estados multinacionales surgen de la incorporación de culturas, que previamente disfrutaban de autogobierno y estaban territorialmente concentradas a un Estado mayor. En el segundo caso, la diversidad cultural surge de la inmigración constante, formando grupos étnicos dentro de un Estado. La respuesta frente a estos dos tipos diferentes de arreglos estatales debe ser completamente diferente.

${ }^{30}$ Kymlicka, Ciudadanía multicultural, p. 76. 
RODRIGO ELIZARRARÁS

Como demandan los Acuerdos de San Andrés, es necesario el replanteamiento del federalismo en este país. Mediante una delimitación de las fronteras políticas con las étnicas se podría evitar gran parte de los problemas derivados de la falta de autoridades propias de los indígenas. La creación de municipios y distritos administrados por autoridades indígenas es algo que este autor respaldaría, así como una reestructuración del federalismo mexicano, buscando compaginar las fronteras políticas con las étnico-culturales.

2) Derechos especiales de representación: implican la representación de las diversas minorías, con el fin de dotar a las instituciones democráticas de una mayor inclusión y representatividad. Algunos países como Nueva Zelanda y Colombia han adoptado este tipo de medidas, reservando un número de escaños en el Congreso para representantes de grupos desfavorecidos.

La representación de indígenas en las Cámaras tanto locales como federales ha de canalizarse a través de los partidos políticos, que no son lo suficientemente incluyentes. Por cierto, tampoco se cuenta con un sistema de cuotas para garantizar la representación de las etnias ante las instituciones estatales. Siguiendo el pensamiento de Kymlicka, 102 sería necesario crear estos mecanismos de representación para garantizar cierta participación de las minorías en los niveles locales y nacionales de gobierno. Muchos piensan que la representación política de autoridades indígenas en las cámaras sólo es una actitud populista, ya que su poder real sería mínimo, rebasado continuamente por la voz de las mayorías.

De los autores escogidos, éste es el único que abiertamente se manifiesta a favor de una defensa cultural de estos pueblos o grupos. "Si respetamos a los Indios como Indios, esto es, como miembros de una comunidad cultural distinta, entonces, debemos reconocer la importancia que para ellos tiene la herencia cultural, y debemos reconocer la legitimidad de las demandas hechas por ellos a favor de la protección de su cultura."31 Para Kymlicka, estos reclamos merecen atención, aún

${ }^{31}$ Kymlicka, Liberalism, Community and Culture, p. 150-151. 
cuando exista un conflicto con algunos de los principios expresados en las leyes o en la Constitución; de ser así es preciso hacer las reformas necesarias para armonizar las diferentes perspectivas en un marco democrático y legal.

La supervivencia de una cultura no está garantizada, y cuando se ve amenazada debemos actuar para protegerla, sobre todo porque las culturas no son valiosas en y por sí mismas, sino porque únicamente mediante la participación en una 'cultura societal' ${ }^{32}$ las personas pueden tener acceso a un conjunto de opciones significativas. Para que esto sea posible, los individuos no sólo necesitan acceso a la información, capacidad para evaluarla reflexivamente y libertad de elección y asociación, sino también estar incorporados en una cultura societal. Ello implica una defensa de las lenguas, las costumbres y las formas propias de gobierno de cada grupo minoritario. Resulta de importancia fundamental permitir la participación en las instituciones gubernamentales mediante su propio idioma.

Un argumento que utiliza para defender los derechos de las minorías étnicas es el de la desigualdad, que mediante la aplicación de condiciones especiales persigue equilibrar la situación de grupos que padecen desventajas. Contribuir "a reducir la vulnerabilidad de los grupos minoritarios ante las presiones económicas y las decisiones políticas del grueso de la sociedad", 33 lo cual implica la instrumentación de derechos diferenciados. Bajo esta luz, los derechos que reclaman los indígenas en los ASA deben de constituirse con miras a reducir la desigualdad en la que viven. No obstante, la experiencia indica que la simple inclusión legislativa de derechos que establezcan una mejor distribución de recursos es insuficiente para garantizarla.

${ }^{32}$ El mundo moderno está dividido, según Kymlicka, en culturas societales, o sea, una cultura que proporciona a sus miembros unas formas de vida significativas a través de todo el abanico de actividades humanas, incluyendo la vida social, educativa, religiosa, recreativa y económica, abarcando las esferas pública y privada.

${ }^{33}$ Kymlicka, Ciudadania multicultural, p. 61. 


\section{RODRIGO ELIZARRARÁS}

Por otro lado, puntualiza que estos derechos diferenciados no deben otorgarse a colectividades sino a individuos. "Los derechos diferenciados en función de grupo pueden ser otorgados a los miembros individuales de un grupo, o al grupo como un todo o a un Estado/provincia dentro del cual el grupo en cuestión constituye la mayoría." ${ }^{34}$ Esto, como ya hemos apuntado, constituye un problema, ya que los ASA demandan derechos otorgables a los pueblos indígenas como entidades colectivas, de los que Kymlicka evita hablar. Situar a la comunidad por encima de los individuos, como pretenden los comunitaristas, es imposible sin romper con el principio de igualdad de derechos y libertades propio del liberalismo, por lo que resulta pertinente la recomendación de efectuar las modificaciones necesarias para que los derechos que se otorguen a los grupos indígenas no estén sujetas a entidades jurídicas colectivas, como pueblos, comunidades o etnias.

Por último, es importante mencionar que una de las mayores críticas a la teoría de Kymlicka, es la referida a la cuestión de lidiar con las minorías iliberales que existen dentro del Estado; por ejemplo, qué hacer cuando estas minorías tienen diferentes concepciones de los roles de género y relegan a las mujeres a posiciones inigualitarias respecto de

104 los hombres dentro de sus comunidades. Según este autor, habría que permitir la existencia de estas minorías no liberales y poco a poco persuadirlas de abandonar sus prácticas. Los liberales, dice, tendrán que aprender a vivir con minorías iliberales dentro de un territorio nacional, de la misma forma como se soporta a países que no son liberales; asimismo asumir la responsabilidad de denunciar la existencia de prácticas opresivas. Se debe apoyar desde fuera a los liberales dentro de estos grupos, con el objetivo de alcanzar la libertad en algún momento. ${ }^{35}$

Con los pensadores anteriores, Kymlicka comparte la idea de que es importante modificar las instituciones para hacer posible la coexistencia de distintos grupos en un espacio común, pero con un arreglo

${ }^{34}$ Idem, p. 72.

${ }^{35}$ Véase Kymlicka, "The Good, the Bad and the Intolerable", en Dissent, summer, 1996. 


\section{LOS ACUERDOS DE SAN ANDRÉS}

equitativo para las partes. "Encontrar respuestas moralmente defendibles y políticamente viables a dichas cuestiones constituye el principal desafio al que se enfrentan las democracias en la actualidad." 36 Precisamente, la resistencia a cambiar las instituciones que lo han mantenido en el poder y con el control de éste, es el mayor empeño del gobierno mexicano. Cada día es más urgente la necesidad de modificar estas estructuras, a menos que se prefiera el paulatino pero inevitable resquebrajamiento del Estado.

${ }^{36}$ Kymlicka, Ciudadania multicultural, p. 13. 